\title{
Nonlinear Absorptions of CdSeTe Quantum Dots under Ultrafast Laser Radiation
}

\author{
Zhijun Chai, Yachen Gao, Degui Kong, and Wenzhi Wu \\ School of Electronic Engineering, Heilongjiang University, Harbin 150080, China \\ Correspondence should be addressed to Yachen Gao; gaoyachen@hlju.edu.cn and Wenzhi Wu; wuwenzhi@hlju.edu.cn
}

Received 7 May 2016; Accepted 20 June 2016

Academic Editor: Yan Wang

Copyright ( 2016 Zhijun Chai et al. This is an open access article distributed under the Creative Commons Attribution License, which permits unrestricted use, distribution, and reproduction in any medium, provided the original work is properly cited.

The oil-soluble alloyed CdSeTe quantum dots (QDs) are prepared by the electrostatic method. The basic properties of synthesized CdSeTe QDs are characterized by UV-Vis absorption spectroscopy, photoluminescence spectroscopy, inductively coupled plasma mass spectrometry, and transmission electron microscope. The off-resonant nonlinear optical properties of CdSeTe QDs are studied by femtosecond $Z$-scan at $1 \mathrm{kHz}$ (low-repetition rate) and $84 \mathrm{MHz}$ (high-repetition rate). Nonlinear absorption coefficients are calculated under different femtosecond laser excitations. Due to the long luminescent lifetime of CdSeTe QDs, under the conditions of high-repetition rate, for open-aperture curve, heat accumulation and bleaching of ground state are responsible for the decrease of two-photon absorption (TPA) coefficient.

\section{Introduction}

Quantum dots (QDs) provide a feasible alternative for electroluminescence light-emitting diode and fluorescence imaging because of their unique photophysical characteristics, such as size tunable photoluminescence (PL) spectrum, high quantum yields, broad absorption, and narrow emission wavelengths [1]. When the nanoparticles are excited by ultrafast laser pulse, nonlinear absorption, luminescence, coherent phonon vibration, and plastic deformation [2] can be predicted and observed. The study of nonlinear optical properties for nanoparticles using $Z$-scan techniques provides complementary information to extend the potential application to optical switching and information storage [3, 4]. This nonlinear optical information can help to understand deeply fundamental properties of nanomaterials including but not limited to linear optical properties. Many experiments as well as theoretical calculations were carried out to investigate the nonlinear optical properties of II-VI QDs, including CdTe [5], CdSe [6], and CdS [7] QDs. Recently alloyed IIVI QDs have been extensively synthesized since their band gap can be tuned without the change of nanoparticle size. Through changing the composition of QDs' element, the band gap can be expanded from ultraviolet to near infrared range, so the nonlinear optical properties are important for the further investigation to alloyed QDs. Now Z-scan measurements have performed on some kinds of alloyed QDs, such as CdSeS/polystyrene film [8], CdSeS/ZnS [9], and $\mathrm{CuInS}_{2}$ QDs [10], due to their unique optoelectronic properties.

Nonlinear absorption, especially TPA, of semiconductor QDs is of current interest toward applications such as nonlinear optical gain media [11], optical limiting [12], or biological labeling/imaging [13]. The higher the cross section per particle or volume, the lower the excitation intensities needed to obtain a high enough response in TPA applications with the same amount of material [14]. II-VI semiconductorbased QDs offer significantly higher TPA cross sections with respect to the particle volume.

In this work we investigated nonlinear absorption of CdSeTe QDs in toluene using femtosecond $Z$-scan technique at $800 \mathrm{~nm}$. CdSeTe QDs are one kind of alloy II-VI semiconductor QDs, which can change the band gap through the change of the molar ratio of Selenium (Se) and Tellurium (Te). The open-aperture $Z$-scan behaviors of the samples are investigated based on the local nonlinear responses including TPA. The results obtained from the experimental $Z$-scan measurements are compared with the influence of repetition rate. With pump-power intensity increasing, TPA is investigated using classical theoretical formula [15]. Finally, 
the origin of nonlinear absorption for CdSeTe QDs is briefly discussed.

\section{Experimental Approach}

2.1. Synthesis. CdSeTe QDs are prepared by injecting of cadmium oxide, oleic acid, Selenium powder, Tellurium powder, trioctylphosphine (TOP), and octadecene into the flask with three necks. Mixed solution keeps on a period of one hour for this solution is cooled to room temperature; then the reaction temperature increases at a rate of $10^{\circ} \mathrm{C}$ per minute to $180 \sim 260^{\circ} \mathrm{C}$. The detailed preparation method was similar to the description in $[16,17]$. Samples of colloidal CdSeTe QDs passivated with organic ligands (octadecene and trioctylphosphine). The organic capping groups sterically stabilize the dots in toluene. Optically thin and transparent, close-packed QDs are deposited on carbon-coated copper grids. High-resolution transmission electron microscope (TEM) measurement is performed on a Tecnai G220 S-Twin microscope operating at a high voltage at $200 \mathrm{kV}$.

2.2. Experimental Setup. The $Z$-scan measurements with low-repetition rate are carried out with femtosecond laser pulses (Coherent, Legend) operating at $800 \mathrm{~nm}$ at a repetition rate of $1 \mathrm{kHz}$ to avoid thermal accumulation. The $Z$-scan measurements with high-repetition rate are performed with seed laser (Spectra Physics, Mai Tai) operating at $800 \mathrm{~nm}$ at a repetition rate of $84 \mathrm{MHz}$ as shown in Figure 1. In the $Z$ scan technique, a sample of thickness $L$ is translated through the focus of a beam with a Gaussian spatial profile, and the transmission through an aperture behind the sample is measured as a function of the longitudinal coordinate $Z$. The linear transmittance of the solution is adjusted to be $50 \%$ at $800 \mathrm{~nm}$ by adding toluene to CdSeTe QDs solution. The incident and transmitted laser powers are monitored as the samples are moving along the propagation direction of the laser pulses. The laser pulses are focused onto a $2 \mathrm{~mm}$ thick quartz cuvette, which contained the CdSeTe QDs solution, with a planoconvex lens (focal length of $200 \mathrm{~mm}$ ). The Gaussian laser beam waist $\omega_{0}$ is measured to be $80 \mu \mathrm{m}$ after focusing with the scanning knife-edge technique. The openaperture Z-scan signals are detected with Silicon detector (Thorlabs, Det36A) and collected by the computer through a lock-in amplifier (EG\&G, Model 5210). In order to testify the validity of femtosecond $Z$-scan experiment, the measurement of carbon sulfide is performed. The nonlinear absorption coefficients of carbon sulfide at femtosecond laser excitation are $\beta=1.5 \times 10^{-2} \mathrm{~cm} / \mathrm{GW}$ at power density of $I_{0}=$ $31 \mathrm{GW} / \mathrm{cm}^{2}$, which is reasonably consistent with the reported results in [18].

\section{Results and Discussion}

3.1. Optical and Structural Characterization. The band gap for bulk CdSe is around $712 \mathrm{~nm}(1.74 \mathrm{eV})$ at room temperature, and the band gap for bulk CdTe is $827 \mathrm{~nm}(1.50 \mathrm{eV})$ [19]. When the size of them is reduced to several nanometers, the band gaps of II-VI semiconductors shift to higher energy due to quantum size confinement, causing a blue shift of the

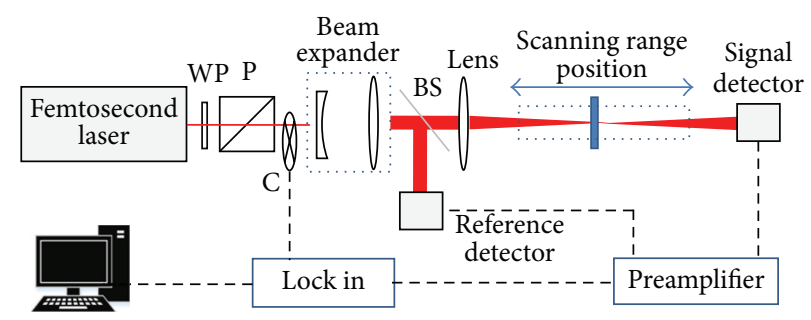

FIGURE 1: Schematic diagram of $Z$-scan setup using femtosecond seed laser as excitation source (high-repetition rate). WP is a halfwave plate, $\mathrm{P}$ is a polarizer, $\mathrm{C}$ is a chopper, and $\mathrm{BS}$ is a beam splitter.

adsorption spectrum. Figure 2(a) shows the UV-absorption and PL spectra of the CdSeTe QDs spectra, which reveals that the size of the nanoparticles is nearly monodispersed represented by the sharp absorption and emission peaks (FWHM $\sim 57 \mathrm{~nm}$ ). The absorption peak can be assigned to excitonic transitions between the lowest electron state $\left(1 \mathrm{~S}_{1 / 2}\right)$ and hole states $\left(1 \mathrm{~S}_{3 / 2}\right)$. The molar ratio of Se and Te in CdSeTe QDs measured is 3:1 using inductively coupled plasma mass spectrometry (ICP-MS). The CdSeTe QDs in Figure 2(b) are basically spherical, sufficiently monodisperse, and well separated, with the average sizes distribution of $3.5 \mathrm{~nm}$ from the observation of TEM image.

3.2. Nonlinear Absorption at Low-Repetition Rate. In the transparent spectral region, the off-resonant nonlinear absorption coefficient can be ascribed multiphoton absorption, free carrier absorption, bleaching, and so on. For the sample, the first absorption peak of nanomaterial usually corresponds to the band gap energy $E_{g}$, where $E_{g}$ is the band gap energy of samples. Nonlinear absorption mainly results from TPA and free carrier absorption in semiconductor materials. Due to the fact that the femtosecond excitation condition of CdSeTe QDs satisfies $\left(E_{g}=2.2 \mathrm{eV}\right)$, TPA can take place. However, the contribution of excitonic emission to the nonlinearity cannot be neglected. Off-resonant optical nonlinearity of a series of CdSe nanocrystallite-doped glasses with CdSe particle radii from 3.5 to $6.5 \mathrm{~nm}$ is measured using Nd:YAG pulsed laser as excitation source [20]. The bound electron nonlinearity of nanometer-sized CdSe is negative and reflects the dominant role of the free carrier absorption which is different from the bulk semiconductors. Therefore, nonlinear optical properties of CdSeTe QDs should also come mainly from the contribution of the quantum confinement. Here, open-aperture $Z$-scan curves are shown in Figure 3, which reveals TPA occurs when photon energy of laser radiation is smaller than $E_{g}$, nonlinear absorption coefficient of CdSeTe QDs is measured to be about $3.1 \times 10^{-2} \mathrm{~cm} / \mathrm{GW}$ under the pump-power intensity from 70 to $175 \mathrm{GW} / \mathrm{cm}^{2}$, and it is ascribed to TPA because the heat accumulation is highly depressed by employing femtosecond laser pulses with very low-repetition rate $[21,22]$.

3.3. Upconversion Luminescent Dynamics at Low-Repetition Rate. Luminescent decay dynamics provides additional major information on photoinduced carriers in CdSeTe 

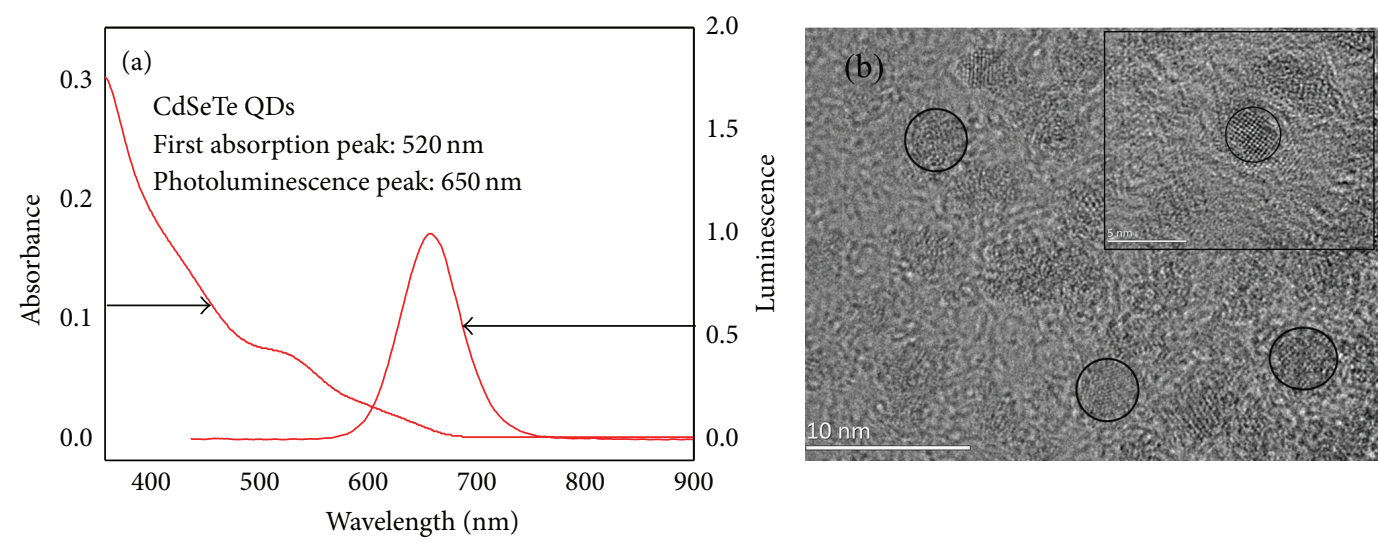

FIgure 2: (a) UV-Vis and PL spectra of CdSeTe QDs samples. (b) TEM image of red-emitting alloyed CdSeTe QDs, where the average size is $3.5 \mathrm{~nm}$. Inset: a high-resolution image displays clear crystal lattice from one of QDs.

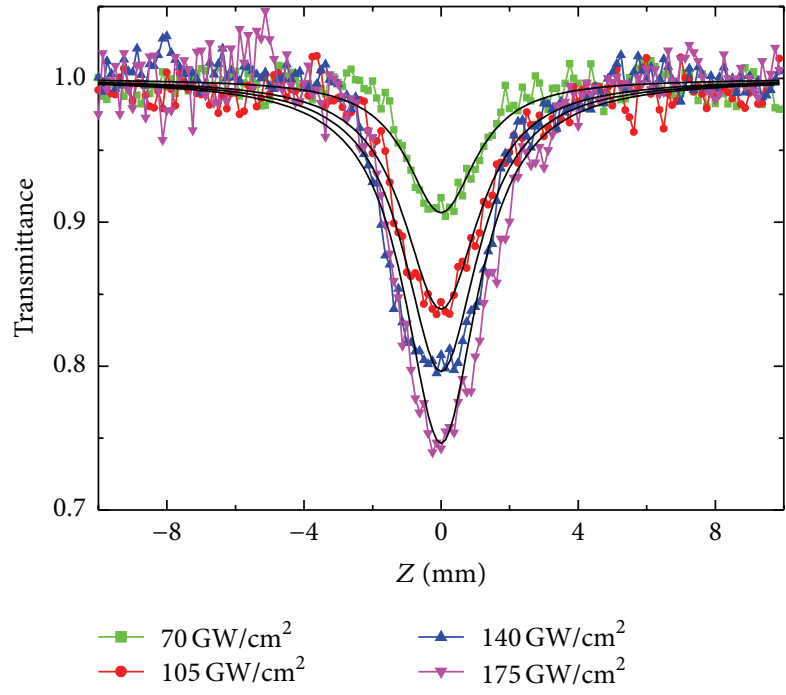

Figure 3: Open-aperture $Z$-scan curves for CdSeTe QD at $800 \mathrm{~nm}$ femtosecond excitation.

QDs. Upconversion luminescence of CdSeTe QDs is easily observed at $800 \mathrm{~nm}$ femtosecond laser excitation. A defectrelated or trapping state is commonly observed in II-VI semiconductor QDs [23]. A broad emission band [24] has been reported and has been attributed to trapping states or surface states. Upconversion luminescence largely ascribes to TPA in nature through a virtual or real intermediate state. Upconversion luminescence decay is not monoexponential and consists of multiple exponential components. The biexponential fitting with convolution strongly implies the involvement of two different states in this upconversion luminescence process [25].

The average PL lifetime values are estimated using the following equation [26]:

$$
\tau_{\mathrm{av}}=\frac{a_{1} \tau_{1}+a_{2} \tau_{2}}{a_{1}+a_{2}}
$$

where $\tau_{\text {av }}$ is the average lifetime, which is used to describe recombination rate of charge carrier for CdSeTe QDs in terms

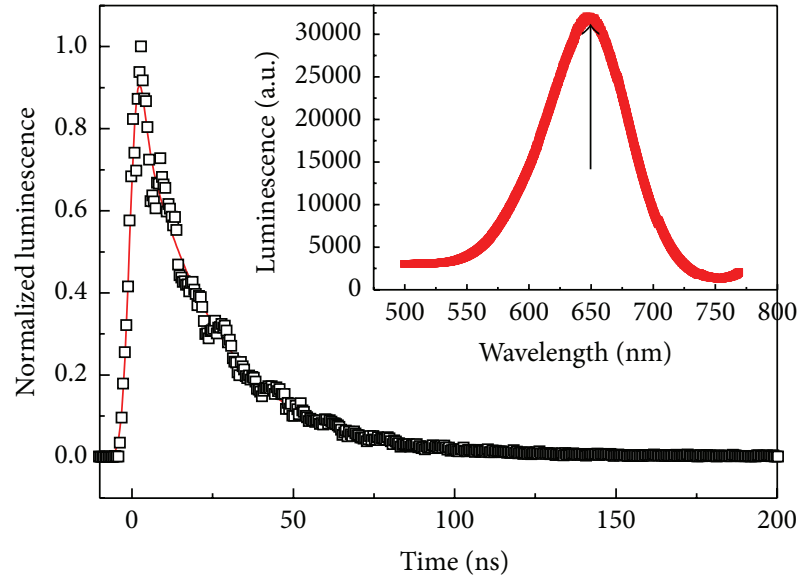

FIGURE 4: Nanosecond luminescent dynamics at the wavelength of the peak at $650 \mathrm{~nm}$.

of a variation in the luminescence decay rates caused by excitonic and trapping states [27]. The average lifetime of $\mathrm{CdSeTe}$ QDs is $23.3 \mathrm{~ns}$ as shown in Figure 4.

3.4. Nonlinear Absorption at High-Repetition Rate. In order to investigate the nonlinear absorption properties, the excitation laser source has been changed as seed laser with highrepetition rate. For high-repetition rate lasers as excitation source, the effects of heat accumulation following the passage of one laser pulse linger for several characteristic thermal time constants. As a result, the thermal effect will increase whenever the time interval between incident laser pulses is much shorter than the characteristic thermal time constant until a steady state is reached between the rates of heat generation and heat diffusion. The repetition rate of the excitation laser source is $84 \mathrm{MHz}$, so the time interval between two pulses is $11.9 \mathrm{~ns}$. As well as we know, alloyed CdSeTe QDs is a typical luminescent nanomaterial. Here the average luminescence lifetime of QDs sample used is about $23.3 \mathrm{~ns}$, so the first laser pulse is used to excite the luminescence of CdSeTe QDs; a variety of carriers are still on the excited state 


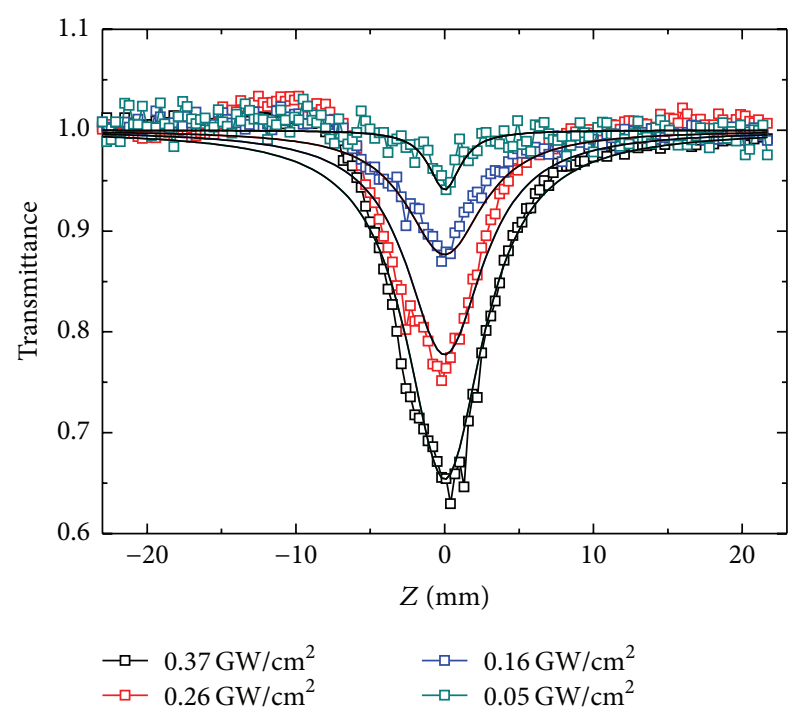

Figure 5: Open-aperture Z-scan curves for CdSeTe QDs at $800 \mathrm{~nm}$ femtosecond excitation.

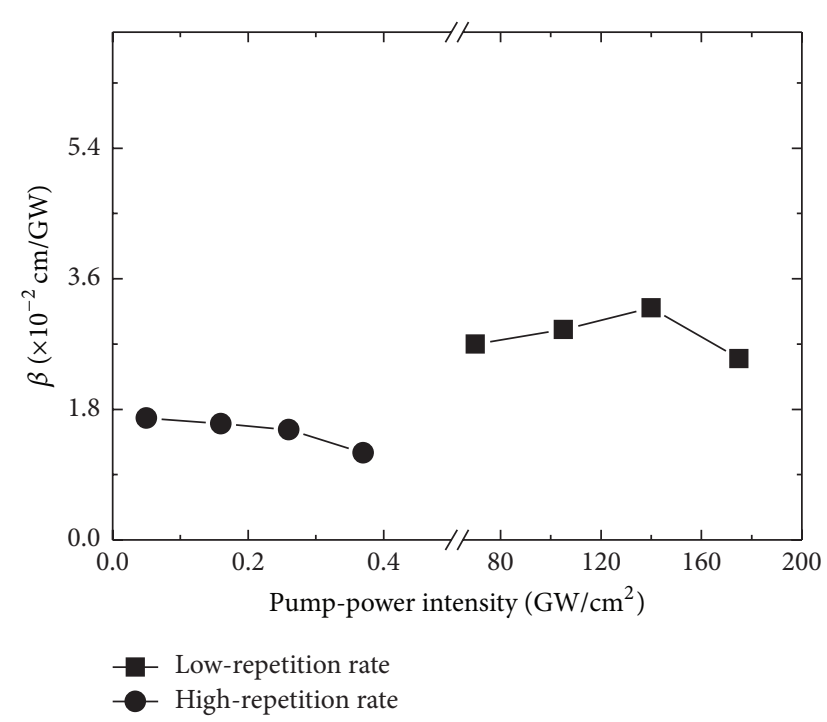

FIGURE 6: TPA coefficient as a function of pump-power intensity of the CdSeTe QDs in toluene.

and not yet back to ground state when the second laser is coming. Open-aperture $Z$-scan curves under different pumppower intensities are shown in Figure 5. The normalized transmittance for the open-aperture condition is calculated from value of $q_{0}(Z)$ and $Z$ [28]. The values of TPA coefficient are calculated by the formula from [29]. Obviously TPA can be affected by the heat accumulation and bleaching of ground state.

3.5. Comparison with the Influence of Repetition Rate. The TPA coefficients of CdSeTe QDs calculated are shown in Figure 6. The values of TPA coefficients of CdSeTe QDs are about $2.4 \times 10^{-2} \mathrm{~cm} / \mathrm{GW}$ which are found to be four and two orders higher in CdSe nanoparticles, respectively, as compared to the bulk CdSe direct and indirect band gap crystal and hence third-order nonlinear susceptibility enhanced in semiconductors QDs [30]. These results obtained are due to the crystalline nature and smaller size of particles. With the pump-power intensities increasing at high-repetition rate, TPA becomes a little smaller. Heat accumulation and the bleaching of ground state can affect the nonlinear absorption coefficient. Ignoring thermal effects in these types of experiments will lead to an erroneous interpretation of the origin and strength of the nonlinearity.

In short, the repetition-rate influence of nonlinear absorption properties for CdSeTe QDs is discussed through the classical theoretical formula. Under the high-repetition rate, heat accumulation due to the fast excitation and bleaching of ground state should be considered. The larger value of third nonlinear optical susceptibility makes them potential candidates for all-optical switching and nonlinear optical devices. Maybe, it is also necessary to consider the laser pump flux in the application of alloyed II-VI QDs to biological and medical imaging.

\section{Conclusions}

In summary, optical nonlinear properties of CdSeTe QDs in toluene are investigated by femtosecond $Z$-scan techniques at low- and high-repetition rate. The properties of TPA are discussed under the consideration of heat accumulation. With the pump intensity increasing, TPA appears and becomes smaller at high-repetition rate. Due to the long luminescent lifetime of CdSeTe QDs, under the conditions of high-repetition rate, for open-aperture curve, the bleaching of ground state and heat accumulation are responsible for the changes of TPA coefficient.

\section{Competing Interests}

All the authors declare that there are no competing interests regarding the publication of this paper.

\section{Acknowledgments}

This research was supported in partial finance by Science Fund of Heilongjiang University for Young Scholars (QL201211, JCL201205), Electronic Engineering Provincial Key Laboratory (P201404), Natural Science Foundation of China (61275117, 61204007), New-Century Training Program Foundation for the Talents by Heilongjiang Province (1254NCET-018), Foundation for University Key Teacher by Heilongjiang University (1252G047), and Heilongjiang Province Postdoctoral Science Foundation (LBH-Q14139).

\section{References}

[1] C.-Y. Zhang and L. W. Johnson, "Quantum dot-based fluorescence resonance energy transfer with improved FRET efficiency in capillary flows," Analytical Chemistry, vol. 78, no. 15, pp. 5532-5537, 2006.

[2] L. Cao and M. Koslowski, "Rate-limited plastic deformation in nanocrystalline Ni," Journal of Applied Physics, vol. 117, no. 24, Article ID 244301, 2015. 
[3] J. R. Heath, "The chemistry of size and order on the nanometer scale," Science, vol. 270, no. 5240, pp. 1315-1316, 1995.

[4] P. Reiss, J. Bleuse, and A. Pron, "Highly luminescent CdSe/ZnSe core/shell nanocrystals of low size dispersion," Nano Letters, vol. 2, no. 7, pp. 781-784, 2002.

[5] Q. H. Jin, W. Z. Wu, Z. R. Zheng et al., "The third-order optical nonlinearity and upconversion luminescence of CdTe quantum dots under femtosecond laser excitation," Journal of Nanoparticle Research, vol. 11, no. 3, pp. 665-670, 2009.

[6] C. A. Leatherdale, W.-K. Woo, F. V. Mikulec, and M. G. Bawendi, "On the absorption cross section of CdSe nanocrystal quantum dots," The Journal of Physical Chemistry B, vol. 106, no. 31, pp. 7619-7622, 2002.

[7] N. Venkatram, D. N. Rao, and M. A. Akundi, "Nonlinear absorption, scattering and optical limiting studies of CdS nanoparticles," Optics Express, vol. 13, no. 3, pp. 867-872, 2005.

[8] X. Li, F. Li, and Z. He, "Large off-resonant and intensitydependent third-order optical nonlinearities of CdSeS quantum dots/polystyrene composite film," Materials Letters, vol. 93, pp. 366-369, 2013.

[9] F. Wu, W. Tian, W. Chen et al., "Optical nonlinearity and optical limiting of CdSeS/ZnS quantum dots," Journal of Modern Optics, vol. 56, no. 17, pp. 1868-1873, 2009.

[10] N. Venkatram, S. K. Batabyal, L. Tian, J. J. Vittal, and W. Ji, "Shape-dependent nonlinear absorption and relaxation in $\mathrm{CuInS}_{2}$ nanocrystals," Applied Physics Letters, vol. 95, no. 20, Article ID 201109, 2009.

[11] B. Guzelturk, Y. Kelestemur, M. Olutas, S. Delikanli, and H. V. Demir, "Amplified spontaneous emission and lasing in colloidal nanoplatelets," ACS Nano, vol. 8, no. 7, pp. 6599-6605, 2014.

[12] G. S. He, K.-T. Yong, Q. Zheng et al., "Multi-photon excitation properties of CdSe quantum dots solutions and optical limiting behavior in infrared range," Optics Express, vol. 15, no. 20, pp. 12818-12833, 2007.

[13] F. Wang, D. Banerjee, Y. Liu, X. Chen, and X. Liu, "Upconversion nanoparticles in biological labeling, imaging, and therapy," Analyst, vol. 135, no. 8, pp. 1839-1854, 2010.

[14] R. Scott, A. W. Achtstein, A. Prudnikau et al., "Two photon absorption in II-VI semiconductors: the influence of dimensionality and size," Nano Letters, vol. 15, no. 8, pp. 4985-4992, 2015.

[15] M. Sheik-Bahae, A. A. Said, T.-H. Wei, D. J. Hagan, and E. W. Van Stryland, "Sensitive measurement of optical nonlinearities using a single beam," IEEE Journal of Quantum Electronics, vol. 26, no. 4, pp. 760-769, 1990.

[16] R. E. Bailey and S. Nie, "Alloyed semiconductor quantum dots: tuning the optical properties without changing the particle size," Journal of the American Chemical Society, vol. 125, no. 23, pp. 7100-7106, 2003.

[17] Z. Pan, K. Zhao, J. Wang, H. Zhang, Y. Feng, and X. Zhong, "Near infrared absorption of $\mathrm{CdSe}_{x} \mathrm{Te}_{1-x}$ alloyed quantum dot sensitized solar cells with more than $6 \%$ efficiency and high stability," ACS Nano, vol. 7, no. 6, pp. 5215-5222, 2013.

[18] S. Couris, M. Renard, O. Faucher et al., "An experimental investigation of the nonlinear refractive index $\left(n_{2}\right)$ of carbon disulfide and toluene by spectral shearing interferometry and $z$-scan techniques," Chemical Physics Letters, vol. 369, no. 3-4, pp. 318-324, 2003.

[19] W. W. Yu, L. H. Qu, W. Z. Guo, and X. G. Peng, "Experimental determination of the extinction coefficient of CdTe, CdSe, and CdS nanocrystals," Chemistry of Materials, vol. 15, no. 14, pp. 2854-2860, 2003.
[20] D. Cotter, M. G. Burt, and R. J. Manning, "Below-band-gap third-order optical nonlinearity of nanometer-size semiconductor crystallites," Physical Review Letters, vol. 68, no. 8, pp. 1200-1203, 1992.

[21] K. Kamada, K. Matsunaga, A. Yoshino, and K. Ohta, “Twophoton-absorption-induced accumulated thermal effect on femtosecond Z-scan experiments studied with time-resolved thermal-lens spectrometry and its simulation," Journal of the Optical Society of America B, vol. 20, no. 3, pp. 529-537, 2003.

[22] S. M. Mian, S. B. McGee, and N. Melikechi, "Experimental and theoretical investigation of thermal lensing effects in modelocked femtosecond Z-scan experiments," Optics Communications, vol. 207, no. 1-6, pp. 339-345, 2002.

[23] S. F. Wuister, F. Van Driel, and A. Meijerink, "Luminescence and growth of CdTe quantum dots and clusters," Physical Chemistry Chemical Physics, vol. 5, no. 6, pp. 1253-1258, 2003.

[24] W. Chen, A. G. Joly, and D. E. Mccready, "Upconversion luminescence from CdSe nanoparticles," Journal of Chemical Physics, vol. 122, no. 22, Article ID 224708, 2005.

[25] W. Z. Wu, Z. R. Zheng, J. P. Zhang et al., "Upconversion luminescence of CdTe nanocrystals by use of near-infrared femtosecond laser excitation," Optics Letters, vol. 32, no. 9, pp. 1174-1176, 2007.

[26] W. Wu, Y. Gao, Q. Chang et al., "Upconversion luminescent characteristics and peak shift of CdSeS nanocrystals under different wavelength laser excitation," Journal of Nanoparticle Research, vol. 13, no. 3, pp. 1049-1061, 2011.

[27] A. L. Rogach, T. Franzl, T. A. Klar et al., "Aqueous synthesis of thiol-capped CdTe nanocrystals: state-of-the-art," The Journal of Physical Chemistry C, vol. 111, no. 40, pp. 14628-14637, 2007.

[28] M. Sharma and S. K. Tripathi, "Two photon absorption studies of PVA coated CdSe/ZnSe and CdSe/CdS core/shell nanostructures," Chemical Physics Letters, vol. 634, Article ID 33029, pp. 266-270, 2015.

[29] G. Tsigaridas, I. Polyzos, P. Persephonis, and V. Giannetas, "A novel approach for analyzing open Z-scan experiments," Optics Communications, vol. 266, no. 1, pp. 284-289, 2006.

[30] A. Gaur, D. K. Sharma, D. S. Ahlawat, and N. Singh, "Multiphoton photoconductivity and optical nonlinearities in $\mathrm{ZnSe}$ and CdSe direct band gap crystals," Journal of Optics A: Pure and Applied Optics, vol. 9, no. 3, pp. 260-264, 2007. 

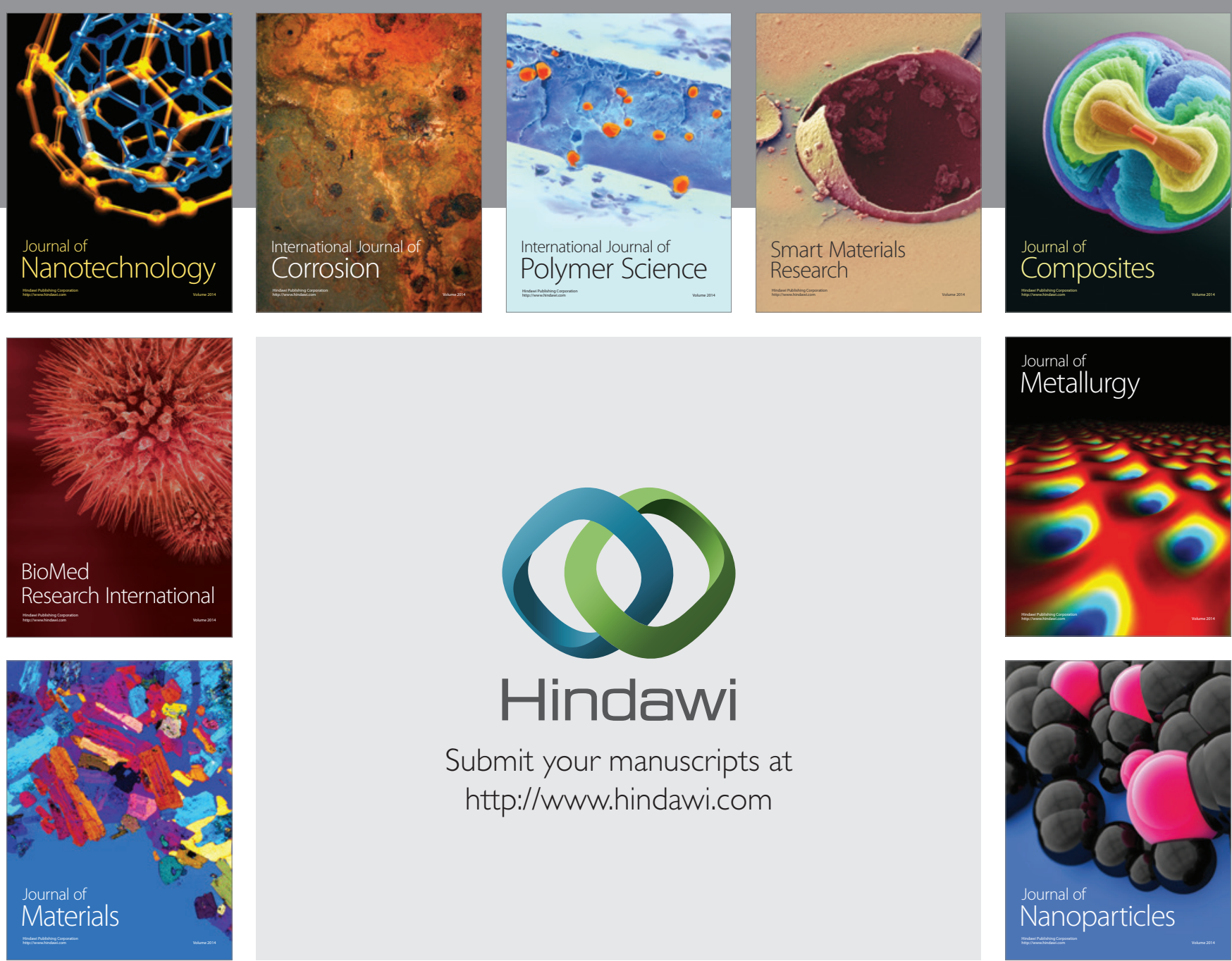

\section{Hindawi}

Submit your manuscripts at

http://www.hindawi.com

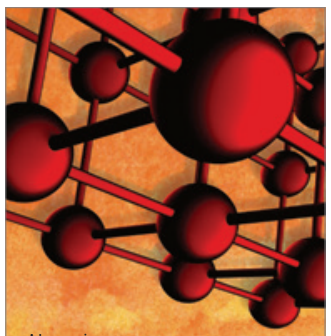

Materials Science and Engineering
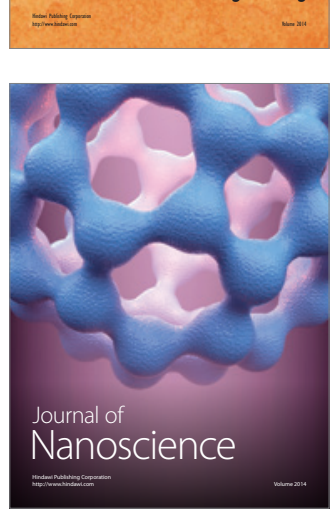
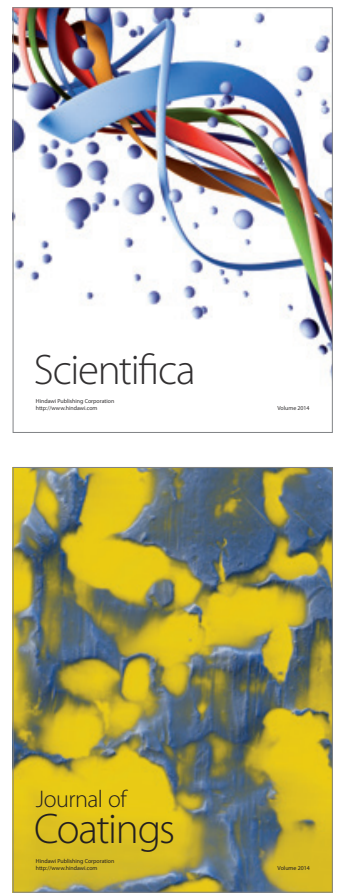
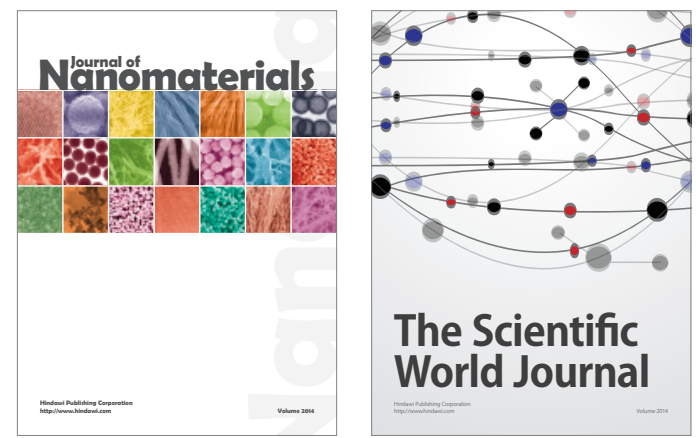

The Scientific World Journal
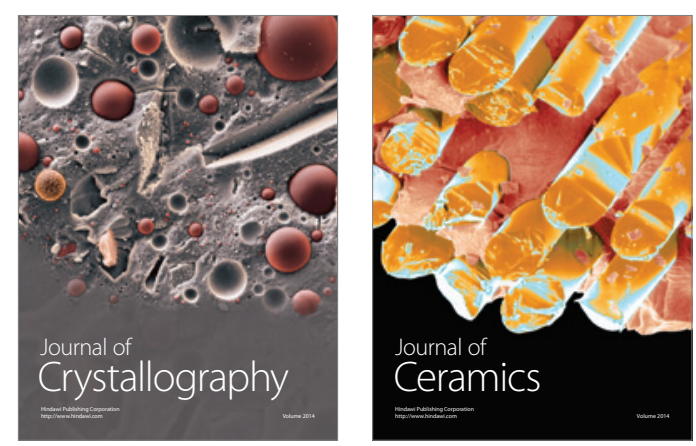
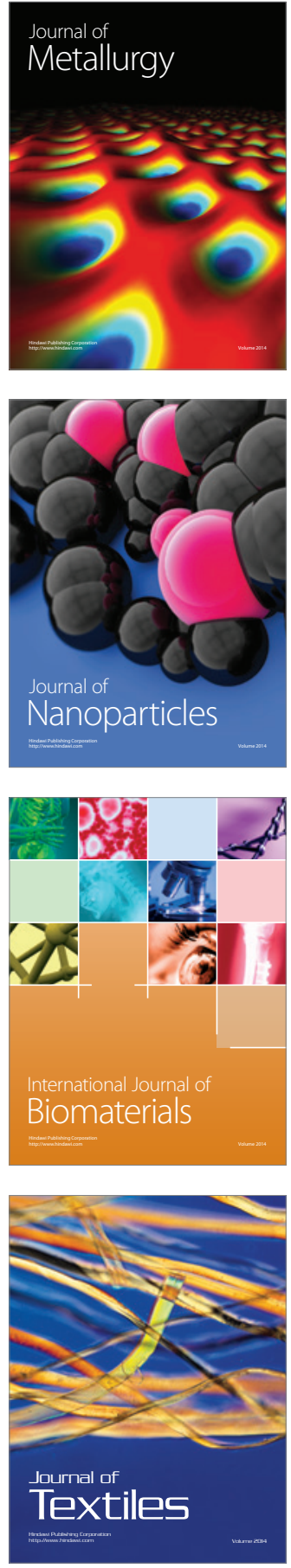